\title{
Fast Unsupervised Seafloor Characterization in Sonar Imagery Using Lacunarity
}

\author{
David P. Williams
}

\begin{abstract}
A new unsupervised approach for characterizing seafloor in side-looking sonar imagery is proposed. The approach is based on lacunarity, which measures the pixel-intensity variation, of through-the-sensor data. No training data are required, no assumptions regarding the statistical distributions of the pixels are made, and the universe of (discrete) seafloor types need not be enumerated or known. It is shown how lacunarity can be computed very quickly using integral-image representations, thereby making real-time seafloor assessments on-board an autonomous underwater vehicle feasible. The promise of the approach is demonstrated on high-resolution synthetic-aperture-sonar imagery of diverse seafloor conditions measured at various geographical sites. Specifically, it is shown how lacunarity can effectively distinguish different seafloor conditions and how this fact can be exploited for target-detection performance prediction in mine-countermeasure operations.
\end{abstract}

Index Terms-Lacunarity, mine countermeasures (MCMs), performance prediction, seafloor characterization, sonar.

\section{INTRODUCTION}

$\mathbf{T}$ HE detection and classification of objects in remotesensing imagery is an important task that is common to many sensor modalities across diverse domains. For underwater mine countermeasure (MCM) applications concerned with detecting mines on the seafloor, there is increasing interest in removing humans from the threat by collecting data with an autonomous underwater vehicle (AUV) equipped with sidelooking sonar.

In sonar imagery, there exists a strong functional relationship between the characteristics of the seafloor and the relative difficulty of detecting objects (e.g., mines). (The generic umbrella term "characteristics" can refer to sediment composition, which affects target to seabed-reverberation levels, as well as seafloor complexity, influenced by topography, rocks, and vegetation.) As a result, knowledge of the seafloor conditions can translate into more accurate predictions of detection performance and more effective automatic target recognition algorithms (e.g., via setting appropriate thresholds for detection and feature extraction algorithms and determining which subsets of training data are most relevant).

However, previous approaches to characterizing the seafloor suffer from various limitations that make them less than ideal for AUV MCM operations. This paper proposes a new fast

Manuscript received September 9, 2014; revised February 23, 2015; accepted May 2, 2015.

The author is with the NATO Science and Technology Organization, Centre for Maritime Research and Experimentation, 19126 La Spezia, Italy (e-mail: williams@cmre.nato.int).

Digital Object Identifier 10.1109/TGRS.2015.2431322 approach to characterizing the seafloor in sonar imagery that overcomes those limitations.

Traditionally, before MCM operations were to commence, sediment samples would be collected in order to estimate the seafloor composition across an entire site, but obtaining the samples requires additional equipment and is at odds with the desire to conduct operations autonomously without human oversight. Moreover, a sediment sample is an intermediate quantity of uncertain importance since it is not necessarily useful for subsequent algorithms that operate on sensor (i.e., sonar) data. For example, it may not matter if flat benign seabed is composed of mud or fine sand if each sediment affects the detection ability (in sonar imagery) in the same manner.

In addition, because seafloor variation often occurs on a very short length scale (of meters), a global estimate of the environment (over a site spanning potentially many square kilometers) based on very few discrete measurement points is typically too coarse to be of value. Instead, there is a need to accurately characterize the seafloor conditions locally, with through-the-sensor sonar data being a logical means.

A common approach to assessing an underwater environment is to perform (hard) segmentation of the seafloor into discrete classes (e.g., sand, mud, and rock). Various supervised classification approaches (requiring labeled training data) have been used to achieve this, with methods employing sets of features based on fractal dimension [1], gray-level co-occurrence matrices [2], spectral energy [3], wavelets [4], or combinations thereof [5]. The major problem with these approaches is that the complete universe of seafloor classes must be known and enumerated a priori. If one surveys a new site and encounters a previously unseen seafloor type, the methods will break down. (In addition, the feature extraction stage of these methods can be computationally intensive, a potential issue for operations in which processing must be performed on-board an AUV.)

Another reason that hard segmentation is unattractive for $\mathrm{MCM}$ is that seafloor conditions often exist on a continuum, rather than fitting tidily into discrete classes. This makes the manually intensive process of compiling ground truth-required for supervised approaches-both laborious and subjective; for example, where does one draw the boundary dividing fine sand from coarse sand? Furthermore, how does one know if such a division is even necessary or warranted?

These arguments drive the desire to characterize each point on the seafloor via a single continuous-valued feature, without resorting to explicit class segmentation. By keeping the characterization problem unsupervised, all classifier training issues can be avoided. Instead, the main challenge becomes to discover a feature that distinguishes seafloor types based on 
how they impact, at a fundamental level, the ultimate quantity of interest-in this paper, object-detection performance.

Some of the earliest attempts to characterize seafloors in sonar imagery were based on trying to fit specific statistical models to the pixel distribution [6], [7]. The learned model parameters (e.g., the shape parameter of a $k$-distribution) would then be used to implicitly characterize the seafloor. The main drawback to this approach is that there is no guarantee that the pixels actually follow the assumed distribution. Although the approach may work well on ideal benign flat seabeds where the parameters are tightly correlated with the sediment (scatterer) size, it is unclear whether the implicit assumptions are satisfied for more complex seafloors, e.g., covered in posidonia (a type of seagrass), characterized by sand ripples, or composed of a mixture of sediments.

Other interesting work [8] has developed a sophisticated sonar texture model, with 15 free parameters, that is derived from the autocorrelation functions (ACFs) of the synthetic aperture sonar (SAS) imaging point spread function and the ACF of the seabed texture sonar cross section. The principal drawbacks to this approach, for our purposes, are the large number of parameters involved in the model and the high computational demands. The former issue introduces complications if a new previously unseen seafloor type is encountered (to be expounded on later), while the latter makes real-time onboard computation untenable.

More recently, features quantifying the anisotropy and complexity of sonar images were introduced [9], [10] as promising ways to characterize the seafloor. These features measure the variation and average, respectively, of filter responses from a family of 2-D Haar-like filters rotated at different angles. Although these features are currently the most appealing solution, their performance is limited. As we will show, among other drawbacks, these features cannot reliably distinguish flat seabed from seabed covered by posidonia. This limitation is notable because it was also shown recently [11] that target-detection performance is a strong function of seafloor conditions, with very different performance resulting from flat seabed and that characterized by posidonia.

In this paper, we instead propose the use of lacunarity, which is a measure of pixel-intensity variation, to characterize seafloors in sonar imagery. Lacunarity has been used successfully for similar environmental assessment purposes in other domains, such as with imagery from synthetic aperture radar (SAR) [12]-[15], hyperspectral [16], [17], and lidar [18] sensors. Despite the previous popularity of lacunarity, we are the first to demonstrate how the quantity can be computed very quickly and efficiently without resorting to any approximations. This is our first contribution, which should be useful for researchers who employ lacunarity to assess textural properties of images in diverse fields - both within and beyond remote sensing - for applications that range from biomedical cancer diagnosis [19] to food inspection [20].

Our second contribution is the demonstration that lacunarity can be applied to the underwater domain for environmental characterization, which has not been shown previously. (Previous work exploiting lacunarity with sonar data used the concept only for anomaly detection, namely, to distinguish pure speckle from regions with structure [21] and to detect objects within sand ripples [22], [23].) Specifically, we show that it is a very powerful quantity for characterizing seafloor to aid targetdetection tasks in side-looking sonar images. This result can be exploited to create object-detection performance prediction models [24], [25] and to inform environmentally adaptive classification algorithms [26]-[28]. Moreover, the results suggest that the lacunarity would be a simple yet powerful feature for performing (hard) seafloor segmentation, if desired.

The remainder of this paper is organized as follows. Section II discusses lacunarity and shows how it can be computed quickly. Section III provides an overview of the measured sonar data used in the experiments, the results of which are reported in Section IV. Concluding remarks and directions for future work are given in Section V.

\section{LACUNARITY}

\section{A. Background}

Lacunarity was originally developed as a way to measure spatial structure in binary-valued data [29], but the concept has since been extended to quantify pixel-intensity variation in grayscale imagery [30]. In this paper, lacunarity is used to characterize the seafloor - and by extension, the minehunting difficulty-in sonar imagery.

The lacunarity of a set of pixels in a grayscale image is the ratio of the variance of the pixel values to the square of the mean of the pixel values. Formally, we define the lacunarity for pixels $x_{i}$ whose indices are in a set $N$ of cardinality $n$ as

$$
L=\frac{\sigma^{2}}{\mu^{2}}
$$

where $\sigma^{2}=\frac{1}{n} \sum_{i \in N}\left(x_{i}-\mu\right)^{2}$ and $\mu=\frac{1}{n} \sum_{i \in N} x_{i}$. However, this can easily be shown to be equivalent to

$$
L=\frac{n \sum_{i \in N} x_{i}^{2}}{\left(\sum_{i \in N} x_{i}\right)^{2}}-1 .
$$

(In [30], the lacunarity is instead defined as $L^{\prime}=L+1$, but the difference is immaterial.) When the set $N$ corresponds to indices that constitute a rectangular block of pixels, this calculation can be done very quickly using integral images.

There is no evidence in the literature that integral-image representations [31] have been exploited for lacunarity calculations in remote-sensing image analysis. Instead, the socalled "gliding window" algorithm [32] has been relied on for the calculation. For example, even a relatively recent paper published in this journal [14] was devoted largely to trying to speed up lacunarity calculations vis-à-vis the gliding window algorithm.

The gliding window algorithm constructs a rectangular window of a specified size, centers it about one pixel, and computes the lacunarity of the pixels that fall within the window. The window center is then shifted to a new pixel, and the lacunarity over the new set of pixels is computed. This process is repeated until the window center has "glided" over the entire image, as 
in a convolution. The end result is effectively a map of the lacunarity around each pixel in the image.

The gross inefficiency of this procedure becomes especially problematic when the lacunarity is to be computed for numerous window sizes - as is commonly desired to establish sizes of structures in images [33] — or when large images are considered. The latter scenario is of particular relevance because a typical MCM survey would consist of thousands of highresolution sonar images, each of which could easily contain millions of pixels. The use of integral images, however, can dramatically speed up the calculation required to compute the lacunarity over any image to such a degree that the computation time is rendered essentially inconsequential.

\section{B. Fast Computation}

An integral image [31] is an image representation that allows for the very fast computation of rectangular Haar-like features at any scale or location in constant time (since the computation does not depend on the size of the input). The construct was formulated in the computer-vision field, where it is used extensively for real-time applications.

Starting from an original (sonar) image $\mathbf{X}$, the corresponding integral image $\mathbf{I}$ is constructed as follows. The value at a location $(r, c)$ in the integral image corresponds to the sum of the pixels above and to the left of $(r, c)$, inclusive, in the original image, $\mathbf{X}$, i.e.,

$$
I(r, c)=\sum_{r^{\prime} \leq r, c^{\prime} \leq c} X\left(r^{\prime}, c^{\prime}\right) .
$$

The integral image is generated using the recursive relation

$$
I(r, c)=I(r-1, c)+z(r, c)
$$

where $z(r, c)$ is the cumulative sum of pixels in a row of the original image

$$
z(r, c)=z(r, c-1)+X(r, c) .
$$

This can be efficiently implemented in MATLAB in a single line of code

$$
I=\operatorname{cumsum}(\operatorname{cumsum}(X, 2), I) .
$$

Next, let $\mathbf{X}^{p}$ indicate the image that results from raising each pixel value to the power $p$, and let $\mathbf{I}_{p}$ denote the associated integral image.

Computing the lacunarity over the sonar image $\mathbf{X}$ requires two integral images, $\mathbf{I}_{1}$ and $\mathbf{I}_{2}$, because of the two sums in (2).

The sum of pixel values in a rectangular area about a given location is computed quickly, with only four array accesses of the integral image, as

$$
\begin{aligned}
S_{p}(r, c)=I_{p}(r- & \alpha, c-\beta)-I_{p}(r-\alpha, c+\beta) \\
& -I_{p}(r+\alpha, c-\beta)+I_{p}(r+\alpha, c+\beta)
\end{aligned}
$$

where $\alpha$ and $\beta$ are the fixed numbers of pixels contained in half of a side (length and width, respectively) of the rectangle. Thus, $\mathbf{S}_{p}$ can be computed for an entire image at once without resorting to any loops.
After computing $\mathbf{S}_{p}$ for $p=1$, 2, the final lacunarity map (associated with the entire sonar image) $\mathbf{L}$ can be quickly computed as

$$
\mathbf{L}=n \frac{\mathbf{S}_{2}}{\mathbf{S}_{1}^{2}}-\mathbf{1}
$$

where $n=4 \alpha \beta$ is the number of pixels summed in the rectangle, the exponentiation and division are performed elementwise, and $\mathbf{1}$ is an appropriately sized matrix of ones.

In this paper, the size of the rectangle used in the lacunarity calculation is $2 \mathrm{~m} \times 2 \mathrm{~m}$ (corresponding to $\alpha=40$ pixels in the along-track direction and $\beta=66$ pixels in the range direction, respectively, for the high-resolution sonar images). This size is chosen to be large enough to capture distinguishing (textural) characteristics of the seafloor but small enough to respect the short length scale at which seafloor conditions are known to vary.

It should also be noted that particular care must be taken to ensure that the precision of the integral-image computations is sufficiently high. If not, errors that can accumulate and grow quickly might manifest [34]. To verify that the precision used is adequate, one can regenerate the original (sonar) image from the integral image via the identity

$$
X(r, c)=I(r-1, c-1)-I(r-1, c)-I(r, c-1)+I(r, c)
$$

and confirm that no errors have been introduced. In this paper, double-precision data types were sufficient.

\section{SONAR DATA SETS}

SAS works by coherently summing received acoustic signals of overlapping elements in an array, and it provides an order-ofmagnitude improvement in resolution over simple (real aperture) side-scan sonar data [35]. The resulting high-resolution SAS imagery provides a detailed view of the seafloor that makes the detection of proud targets possible. In practice, the platform on which the sonar data is usually collected is an AUV.

Typically, a target (e.g., mine) in SAS imagery will exhibit a characteristic highlight-shadow pattern that corresponds to a strong signal return followed in range by a very weak return; the highlight is the result of the echo from the target itself, while the acoustic shadow that is cast is due to the geometry between the target (and specifically its height above the seafloor) and the grazing angle of the transmitted signal.

Mine detection is more challenging in sonar-image regions where shadows (low pixel intensities) and highlights (high pixel intensities) caused by seafloor features are present, as these elements can obscure target responses. In contrast, low pixelintensity variation is essentially another way to describe a benign seafloor where target detection is relatively easy. (Implicit in all of this work is the assumption that the full swath of the sonar image being considered is of sufficient quality (e.g., not suffering from a very low signal-to-noise ratio at long ranges).) These facts suggest that lacunarity would be an appropriate way to characterize the seafloor, and, in turn, the minehunting difficulty, in sonar imagery. In addition, lacunarity is attractive for MCM operations because it can be computed quickly onboard an AUV that possesses very limited processing power. 


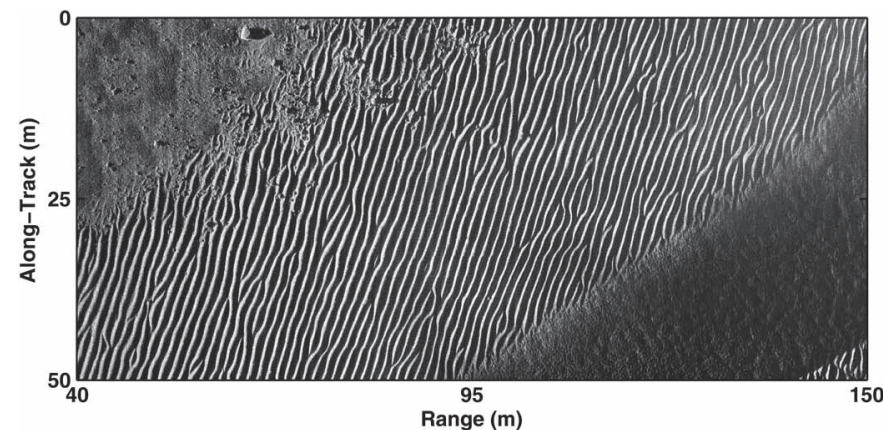

(a)

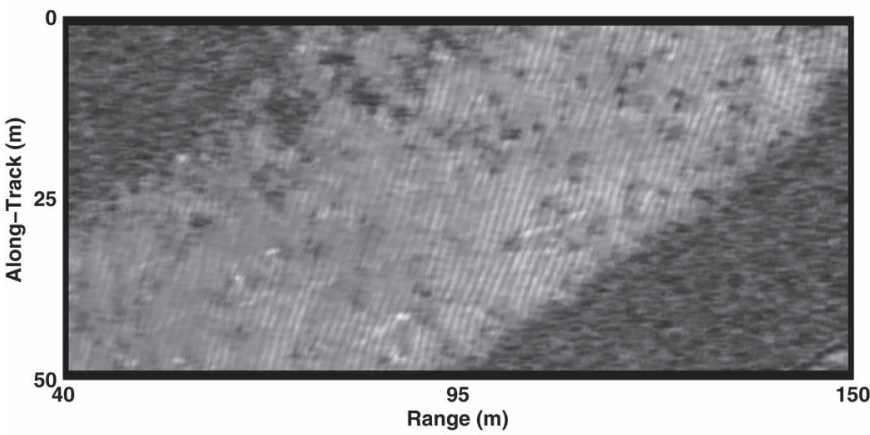

(c)

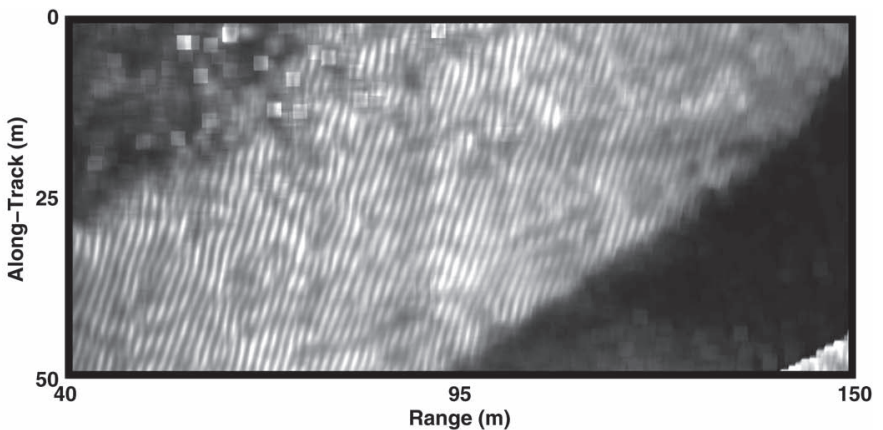

(b)

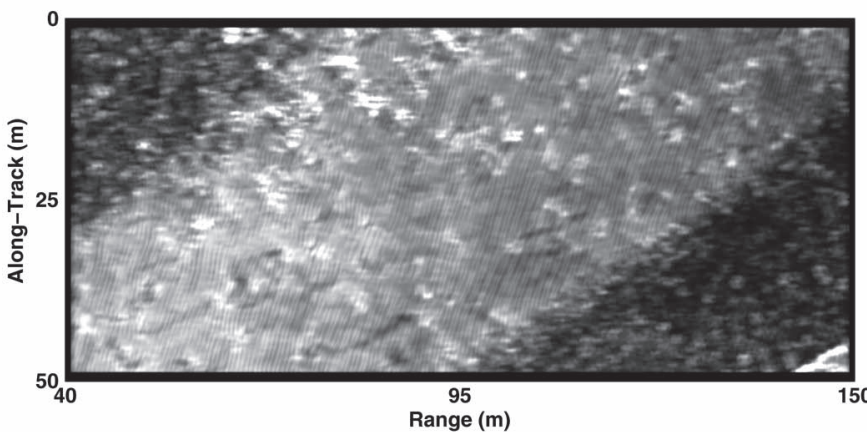

(d)

Fig. 1. (a) SAS image A from near Liepāja, Latvia, that is marked by significant sand ripples and the corresponding (b) lacunarity map, (c) anisotropy map, and (d) complexity map. The ranges of the color scales match the ranges shown in Fig. 7, with lighter shades indicating larger values.

The feasibility of using lacunarity for seafloor characterization is evaluated in this paper using real measured sonar data collected at sea. The primary set of data used in this paper was collected by the SAS-equipped AUV called MUSCLE that is owned by the Centre for Maritime Research and Experimentation (CMRE). The center frequency of the SAS is $300 \mathrm{kHz}$, and the bandwidth is approximately $60 \mathrm{kHz}$. The system enables the formation of high-resolution sonar imagery with a theoretical across-track resolution of $1.5 \mathrm{~cm}$ and a theoretical along-track resolution of $2.5 \mathrm{~cm}$. A standard SAS image from this system comprises over 14 million pixels; an example image is shown in Fig. 1(a). From the figure, it can be observed how the underwater environment can undergo dramatic seafloor changes, e.g., from flat sand to sand ripples, on short length scales.

The images considered in this lacunarity study were drawn from data collected during eight major sea experiments that were conducted by NURC/CMRE between 2008 and 2013 in wide-ranging geographical locations. Specifically, a representative set of five images that collectively span diverse seafloor conditions (sand ripples, posidonia, coarse sand, fine sand, and mud) at various geographical sites (Elba and La Spezia, Italy; Liepāja and Rīga, Latvia; Cartagena, Spain) was selected; these SAS images will be shown in Section IV-B.

\section{EXPERIMENTAL RESULTS}

The feasibility of using lacunarity for seafloor characterization in sonar imagery is assessed in six ways: 1) computation time; 2) qualitative discrimination ability; 3) quantitative discrimination ability; 4) applicability to side-scan sonar imagery; 5) target-detection performance prediction; and 6) ability to handle previously unseen seafloor conditions.
TABLE I

Average COMPutation TIME PER SONAR IMAgE

\begin{tabular}{|c||c|}
\hline FEATURE [METHOD] & TIME (SEC.) \\
\hline \hline LACUNARITY [INTEGRAL IMAGES] & $1.1691 \pm 0.0679$ \\
\hline LACUNARITY [GLIDING WINDOWS] & $3.1247 \times 10^{3} \pm 17.0017$ \\
\hline ANISOTROPY AND COMPLEXITY & $48.2373 \pm 0.2784$ \\
\hline
\end{tabular}

\section{A. Computation Time}

The lacunarity maps for the five SAS images used in the study were computed using two different approaches: the proposed integral-image formulation and the traditional gliding window implementation. The two approaches produce identical results, as no approximations are employed. The anisotropy and complexity maps, as defined in [10], for the images were computed as well, as these features represent the best extant competing approach for our purposes. (The requisite filters for computing those features are considered at 12 different orientations (in $15^{\circ}$ increments).)

All algorithms were implemented in MATLAB and executed on a desktop computer with a 3-GHz Intel Core2 Quad CPU and 8 GB of RAM. (It should be noted that using a lower-level programming language, such as $\mathrm{C}$, would likely result in less dramatic time differences between the lacunarity approaches in the following.) Each of the five SAS images comprised over 14 million pixels. The average computation times (mean \pm standard deviation) required to obtain the resulting maps per sonar image are shown in Table I. (To improve robustness, the timing experiment was repeated five times for each of the five SAS images.)

As can be seen from the table, the integral-image formulation is several orders of magnitude faster than the gliding window approach (about $1 \mathrm{~s}$ compared to more than $52 \mathrm{~min}$ ). The integralimage lacunarity approach is also considerably faster than the 


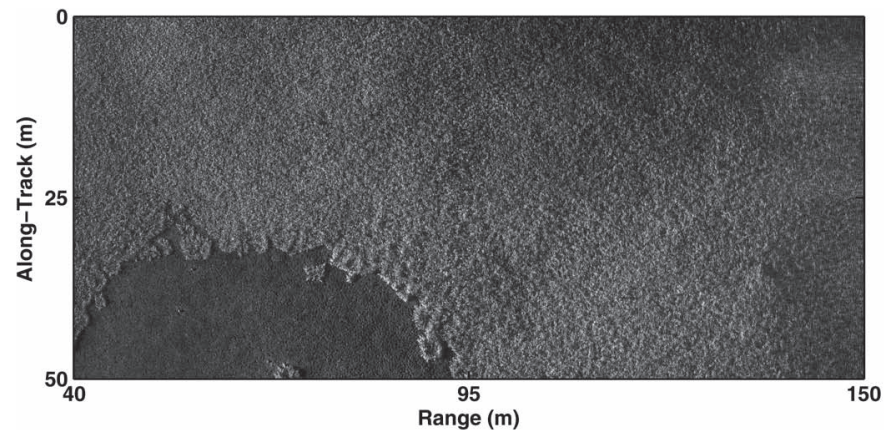

(a)

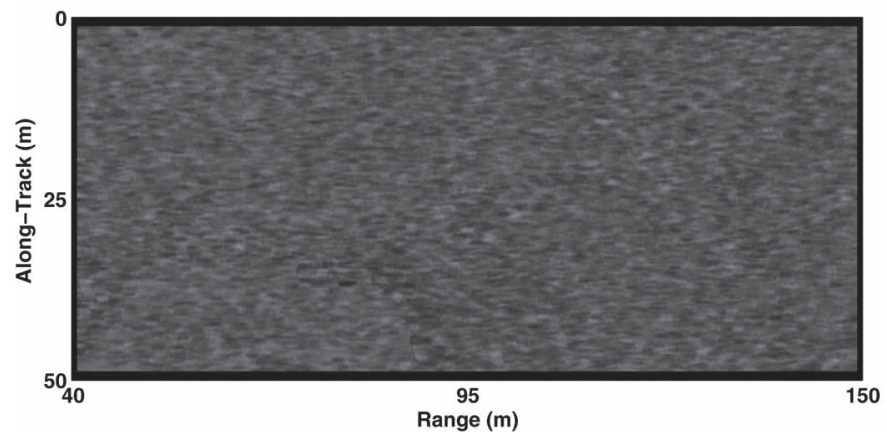

(c)

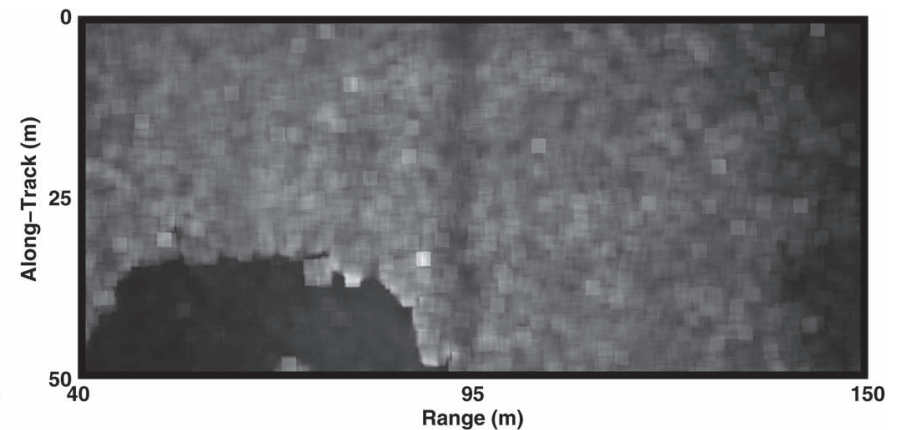

(b)

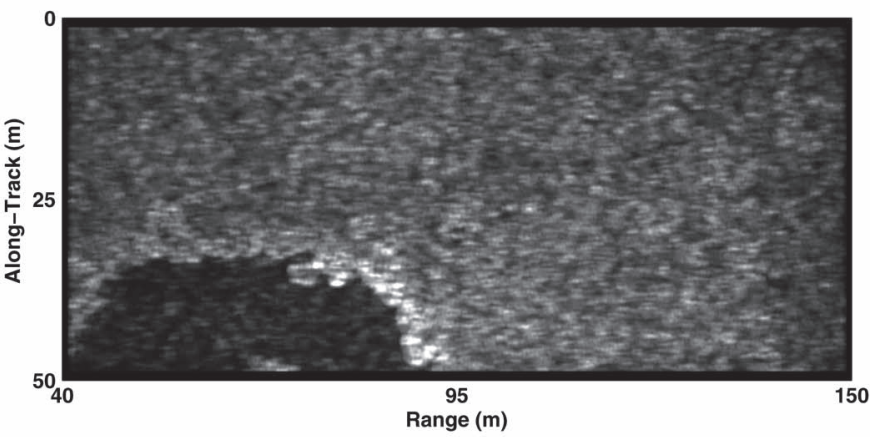

(d)

Fig. 2. (a) SAS image B from near Elba, Italy, that is marked by significant posidonia and the corresponding (b) lacunarity map, (c) anisotropy map, and (d) complexity map. The ranges of the color scales match the ranges shown in Fig. 7, with lighter shades indicating larger values.

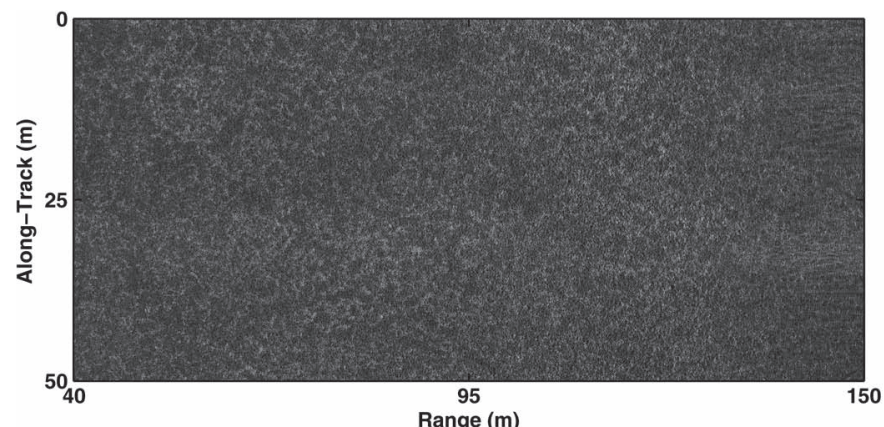

(a)

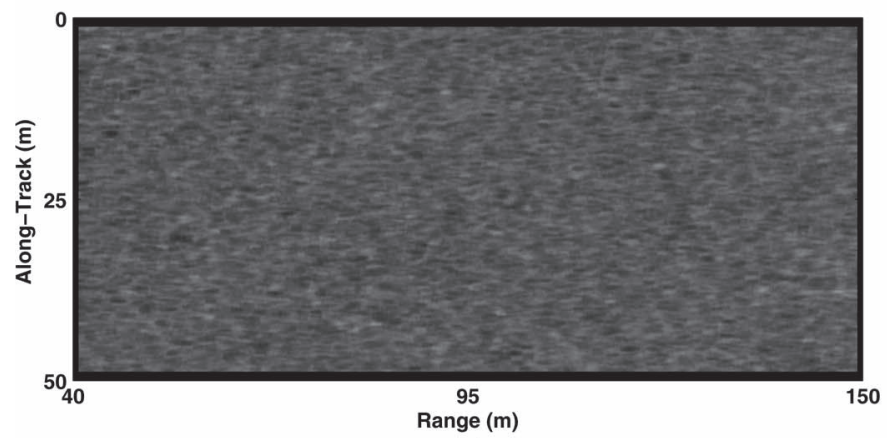

(c)

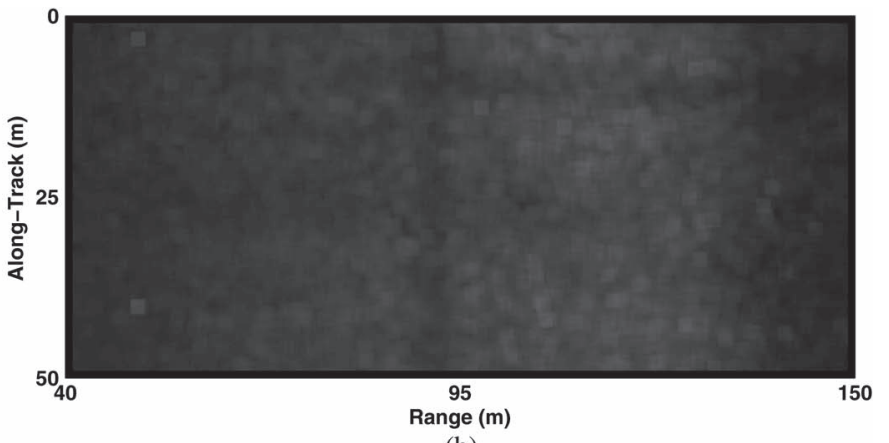

(b)

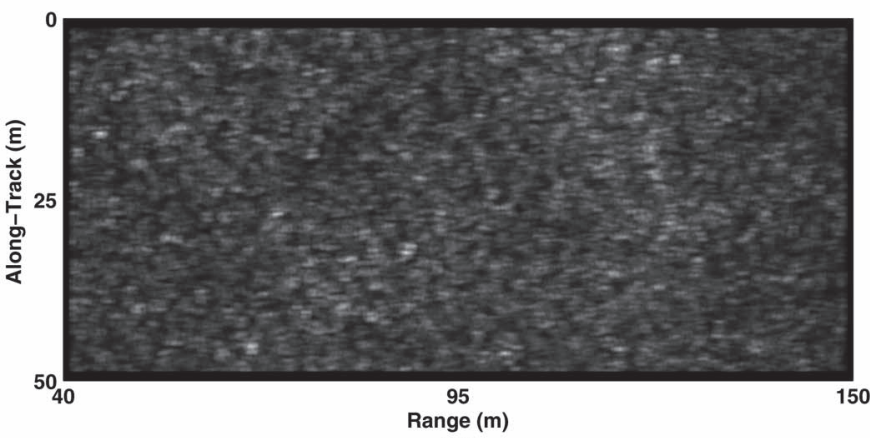

(d)

Fig. 3. (a) SAS image C from near La Spezia, Italy, that is characterized by coarse sand and the corresponding (b) lacunarity map, (c) anisotropy map, and (d) complexity map. The ranges of the color scales match the ranges shown in Fig. 7, with lighter shades indicating larger values.

method computing anisotropy and complexity, further making the former an attractive candidate for use in autonomous operations at sea. To highlight the (delayed) real-time potential of the method, the survey time during which the AUV is collecting the raw data for the 14-million-pixel image is about $30 \mathrm{~s}$.

\section{B. Qualitative Assessment: Environmental Characterization}

The aim of this paper is to assess whether lacunarity can implicitly characterize the seafloor environment in sonar images so that the relative challenge for minehunting can be estimated. 


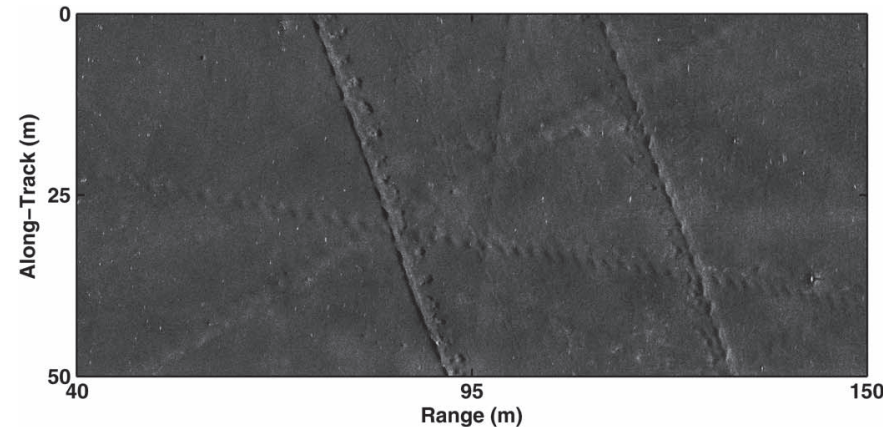

(a)

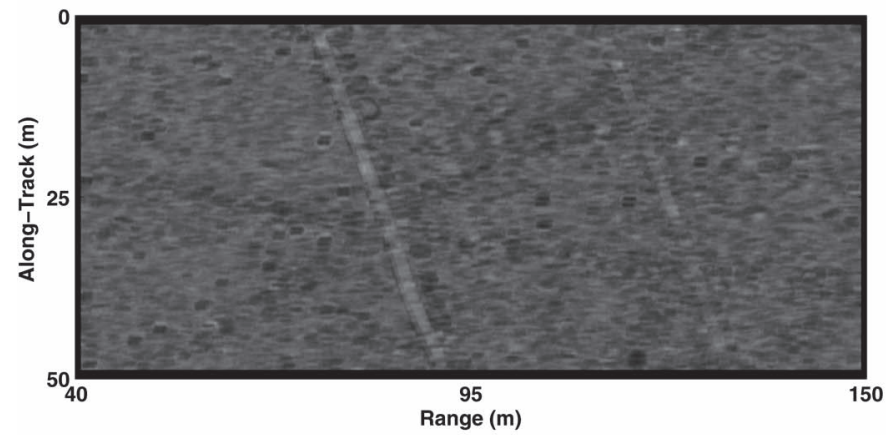

(c)

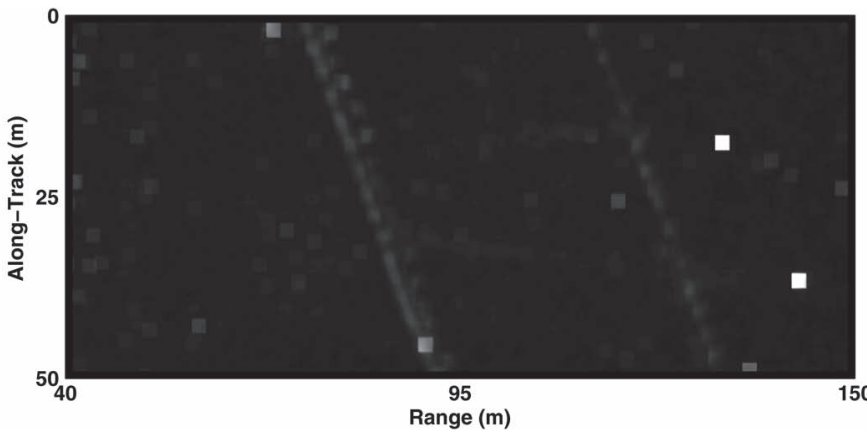

(b)

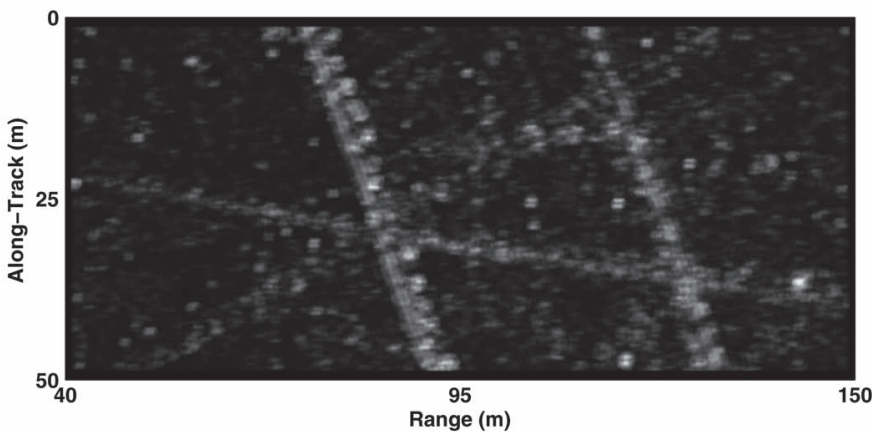

(d)

Fig. 4. (a) SAS image D from near Rīga, Latvia, that is characterized by mud and the corresponding (b) lacunarity map, (c) anisotropy map, and (d) complexity map. The ranges of the color scales match the ranges shown in Fig. 7, with lighter shades indicating larger values.

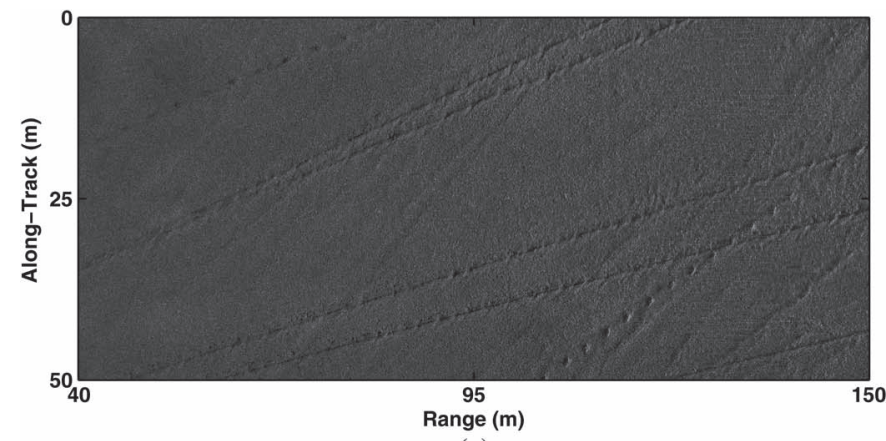

(a)

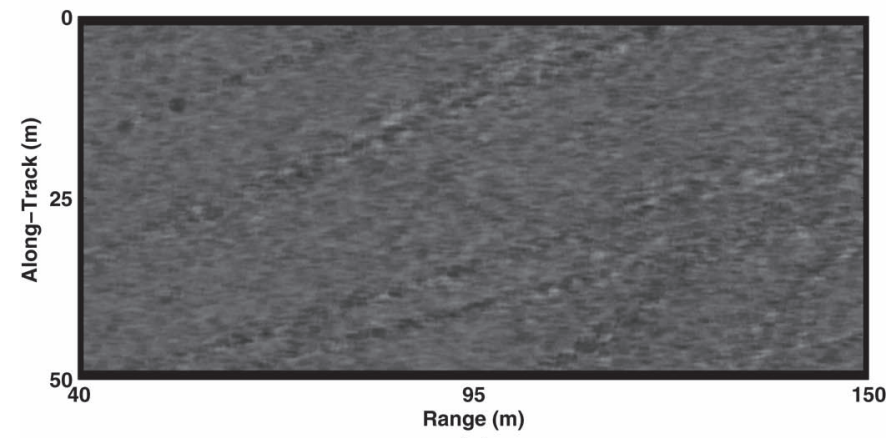

(c)

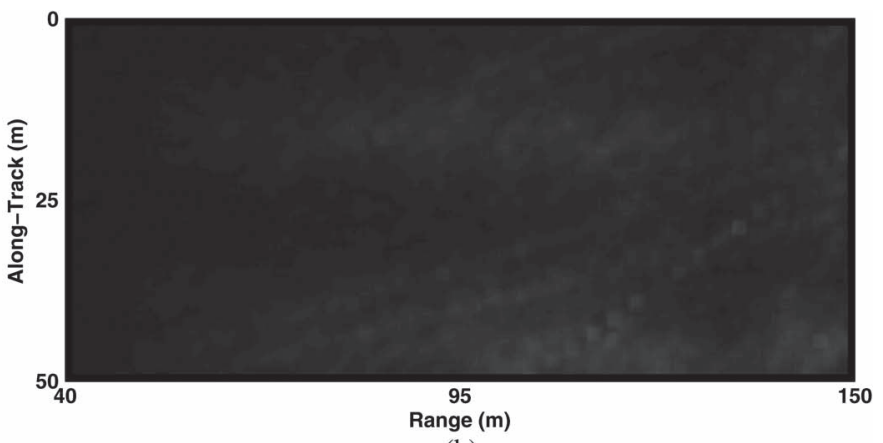

(b)

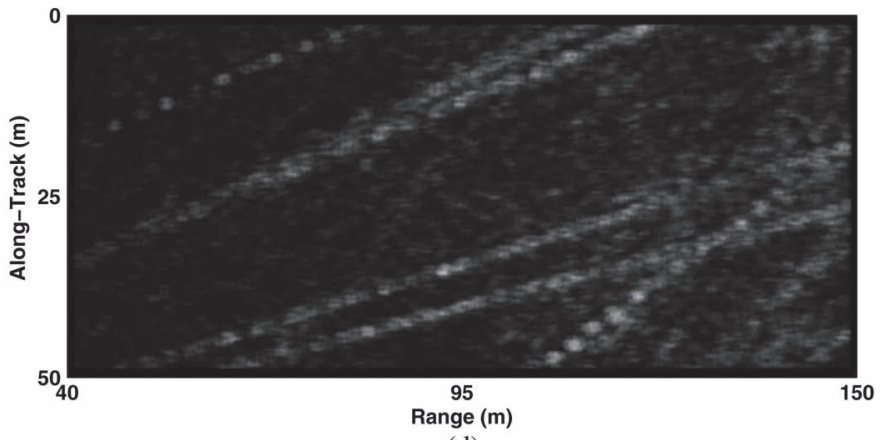

(d)

Fig. 5. (a) SAS image E from near Cartagena, Spain, that is characterized by fine sand and the corresponding (b) lacunarity map, (c) anisotropy map, and (d) complexity map. The ranges of the color scales match the ranges shown in Fig. 7, with lighter shades indicating larger values.

Because this objective is qualitative in nature, we explicitly show the maps of the lacunarity, anisotropy, and complexity computed for each of the five SAS images in Figs. 1-5. (To avoid image-edge effects, the features are not calculated for pixels in a 1-m band around the perimeter of each image.)

It can be seen in the figures that the lacunarity appears to implicitly distinguish sand ripples (Fig. 1), posidonia (Fig. 2), and flat seabed (Figs. 3-5) from each other. Of additional interest is the promising result that a benign seafloor produces somewhat similar lacunarity values regardless of whether it is composed of coarse sand (Fig. 3), fine sand (Fig. 5), or mud (Fig. 4). The ability of the anisotropy and complexity to achieve these two desirable results is less certain. (A more quantitative assessment of the results appears in the next section.) 


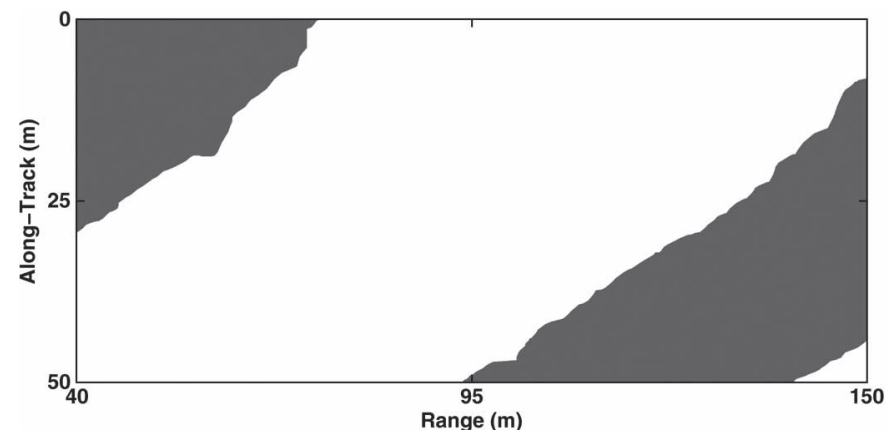

(a)

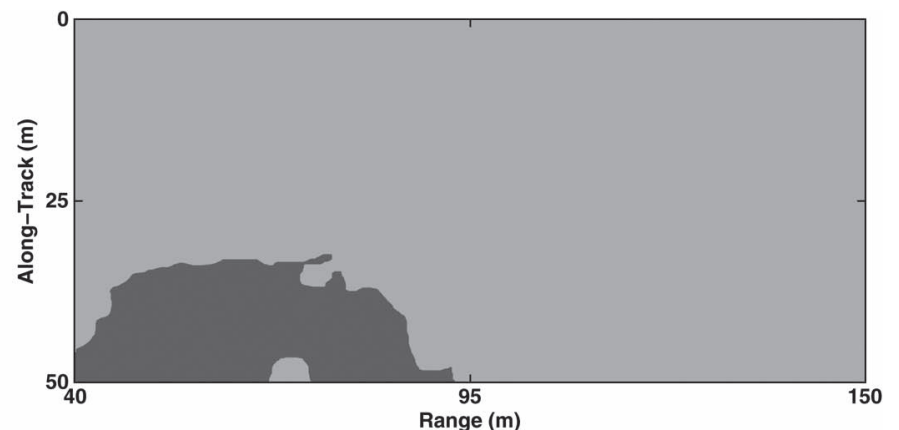

(b)

Fig. 6. Seafloor-class ground truth for (a) SAS image A and (b) SAS image B. White indicates ripples, light gray indicates posidonia, and dark gray indicates flat seabed. (The ground truth for SAS images C, D, and E is unary: flat seabed.)

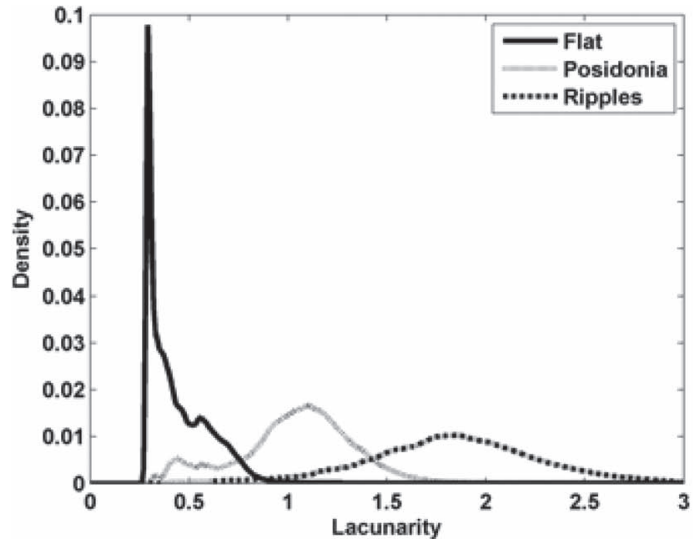

(a)

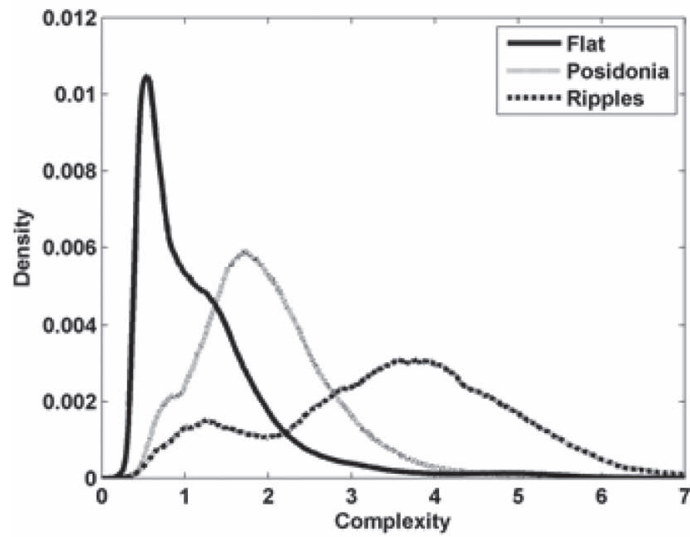

(c)

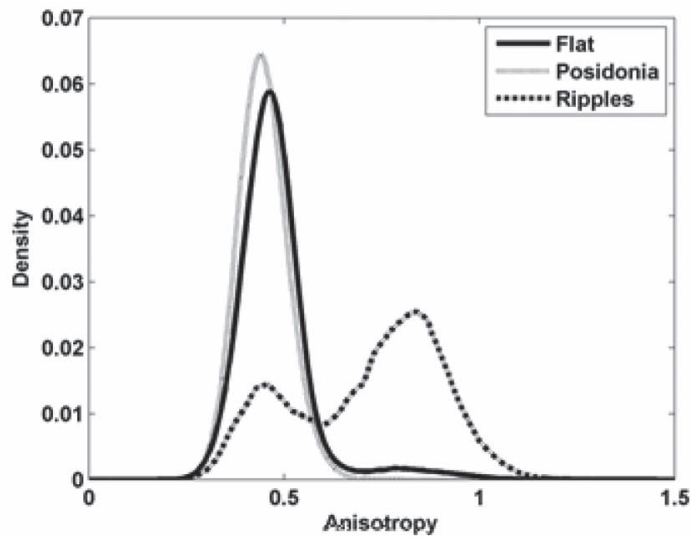

(b)

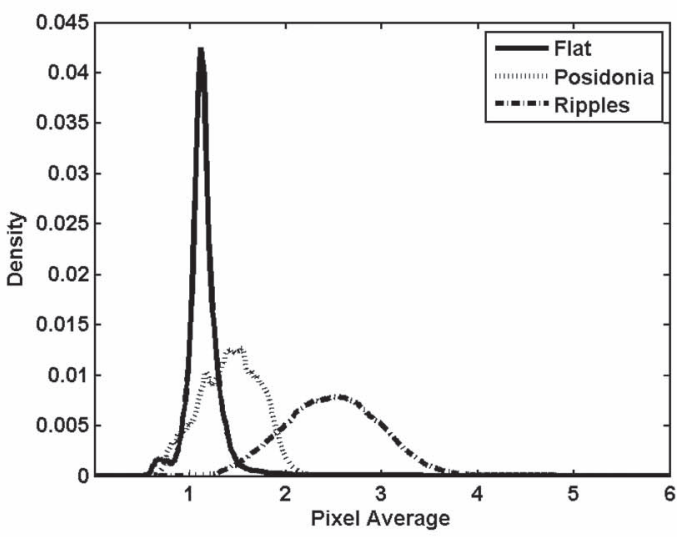

(d)

Fig. 7. Empirical class-conditional probability densities, computed from the five images, of (a) lacunarity, (b) anisotropy, (c) complexity, and (d) pixel average.

\section{Quantitative Assessment: Seafloor Segmentation}

The intention is to use lacunarity to obtain a continuous measure by which the minehunting difficulty of various areas can be rapidly estimated in situ. The goal is not to perform hard segmentation of the seafloor into discrete classes. Nevertheless, to provide quantitative evidence that the lacunarity is a more useful feature for discriminating different seafloor types, we consider the segmentation problem here.

To do so, ground truth indicating the true seafloor class (type) for each pixel in the SAS images is required. The ground truth, created manually, is shown in Fig. 6. With this available, we can construct the empirical class-conditional probability density of each feature (lacunarity, anisotropy, and complexity) for each class, obtained from the five SAS images and shown in Fig. 7.

We also compute the empirical class-conditional probability density for a fourth feature that is simply the pixel average (computed over a $2 \mathrm{~m} \times 2 \mathrm{~m}$ box centered at the given pixel); this is also shown in Fig. 7. This simple feature is included to demonstrate that the pixel intensity alone cannot distinguish the seafloor types, something not readily evident from visually examining the SAS images.

In the ideal case, the density of each class would be well separated from all others, with no overlap. To effect a hard segmentation of the pixels, each pixel is assigned to the seafloor 


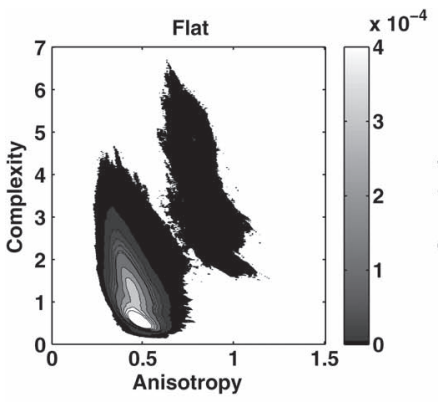

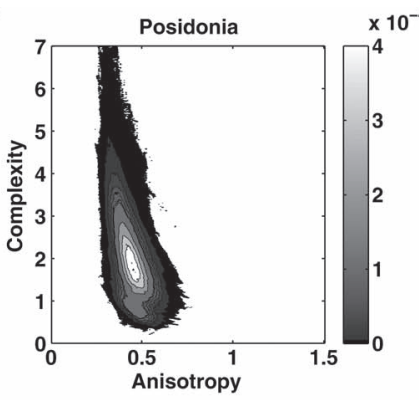

(a)
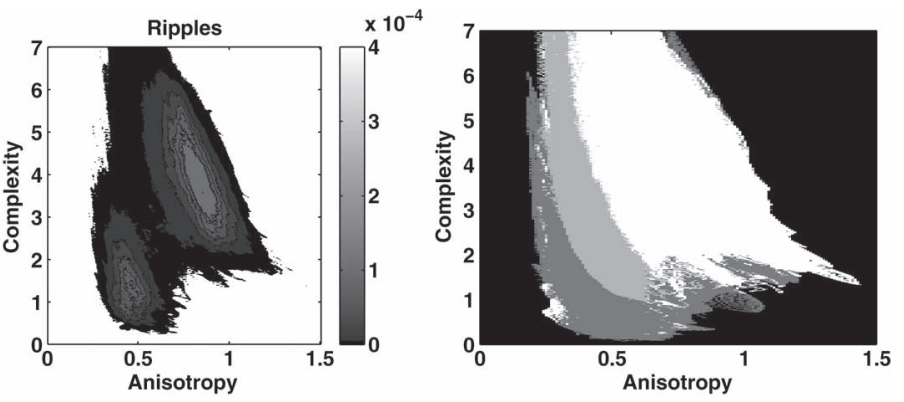

(b)

Fig. 8. (a) Contour plots of the 2-D empirical class-conditional probability densities, computed from the five images, and (b) the optimal class assignments in anisotropy-complexity space (where class colors match those used in Fig. 6; black indicates no data).

TABLE II

CONFUSION MATRIX USING LACUNARITY

\begin{tabular}{|c||c|c|c|}
\hline \multicolumn{1}{|c||}{} & \multicolumn{4}{c|}{ PREDICTED ClASS } \\
GROUND TRUTH & FLAT & POSIDONIA & RIPPLES \\
\hline \hline FLAT & $\mathbf{0 . 9 5 9 1}$ & 0.0383 & 0.0026 \\
\hline POSIDONIA & 0.1637 & $\mathbf{0 . 7 6 1 7}$ & 0.0746 \\
\hline RIPPLES & 0.0169 & 0.1511 & $\mathbf{0 . 8 3 2 0}$ \\
\hline
\end{tabular}

TABLE III

CONFUSION MATRIX USING ANISOTROPY

\begin{tabular}{|c||c|c|c|}
\hline \multicolumn{1}{|c||}{} & \multicolumn{4}{c|}{ PREDICTED ClASS } \\
GROUND TRUTH & FLAT & POSIDONIA & RIPPLES \\
\hline \hline FLAT & $\mathbf{0 . 3 9 7 1}$ & 0.5308 & 0.0721 \\
\hline POSIDONIA & 0.3186 & $\mathbf{0 . 6 6 3 1}$ & 0.0184 \\
\hline RIPPLES & 0.1265 & 0.1576 & $\mathbf{0 . 7 1 6 0}$ \\
\hline
\end{tabular}

TABLE IV

CONFUSION MATRIX USING COMPLEXITY

\begin{tabular}{|c||c|c|c|}
\hline \multicolumn{1}{|c||}{} & \multicolumn{4}{c|}{ PREDICTED ClASS } \\
GROUND TRUTH & FLAT & POSIDONIA & RIPPLES \\
\hline \hline FLAT & $\mathbf{0 . 6 8 2 3}$ & 0.2650 & 0.0527 \\
\hline POSIDONIA & 0.2175 & $\mathbf{0 . 6 4 1 6}$ & 0.1410 \\
\hline RIPPLES & 0.0975 & 0.1990 & $\mathbf{0 . 7 0 3 5}$ \\
\hline
\end{tabular}

TABLE V

CONFusion Matrix Using BOTH ANISOTROPY AND COMPLEXITY

\begin{tabular}{|c||c|c|c|}
\hline \multicolumn{1}{|c||}{} & \multicolumn{3}{c|}{ PREDICTED ClASS } \\
GROUND TRUTH & FLAT & POSIDONIA & RIPPLES \\
\hline \hline FLAT & $\mathbf{0 . 7 5 3 7}$ & 0.1968 & 0.0495 \\
\hline POSIDONIA & 0.2238 & $\mathbf{0 . 7 6 6 6}$ & 0.0096 \\
\hline RIPPLES & 0.1064 & 0.1215 & $\mathbf{0 . 7 7 2 2}$ \\
\hline
\end{tabular}

class for which the density (i.e., likelihood) is a maximum at the feature value of the pixel.

The 2-D probability density when considering anisotropy and complexity jointly is also computed; the resulting densities and the maximum-likelihood class-assignment map are shown in Fig. 8. (In practice, one would not know the exact densities and, hence, the ideal class decision boundaries, so the classification performance that results here is an upper bound. The overly complicated decision boundaries for the 2-D case suggest that its upper bound would not be tight.)

With each pixel of the five SAS images classified in this manner, segmentation performance can be computed for each of the five feature sets considered. The classification results are presented as confusion matrices in Tables II-VI. These confusion matrices are based on over 132 million pixels (approximately 98 million, 24 million, and 9 million pixels in the flat, posidonia, and ripple classes, respectively).
TABLE VI

Confusion Matrix Using Pixel Average

\begin{tabular}{|c||c|c|c|}
\hline \multicolumn{1}{|c||}{} & \multicolumn{4}{c|}{ PREDICTED CLASS } \\
GROUND TRUTH & FLAT & POSIDONIA & RIPPLES \\
\hline \hline FLAT & $\mathbf{0 . 8 9 4 6}$ & 0.1014 & 0.0040 \\
\hline POSIDONIA & 0.3785 & $\mathbf{0 . 5 8 5 6}$ & 0.0359 \\
\hline RIPPLES & 0.0468 & 0.1199 & $\mathbf{0 . 8 3 3 3}$ \\
\hline
\end{tabular}

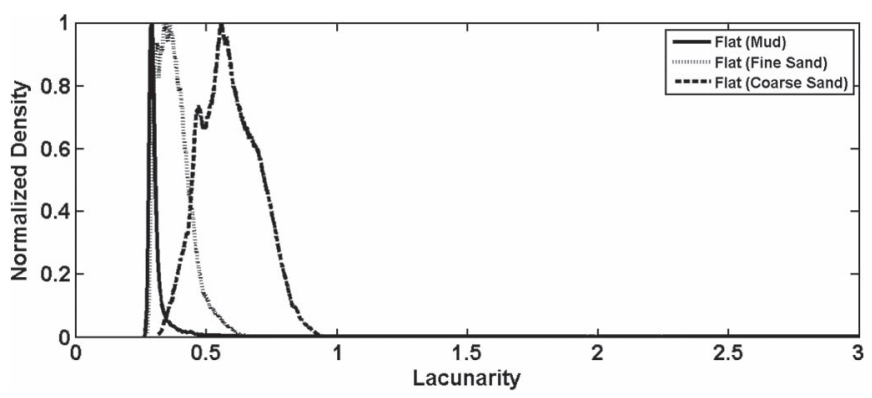

Fig. 9. Empirical probability density of lacunarity, computed from the flat seabed SAS images C (coarse sand), D (mud), and E (fine sand).

From the tables, it can be observed that the lacunarity is quantitatively more accurate than either of the other two features (anisotropy and complexity) alone, as well as the two features considered jointly. (However, it should be noted that the difference between the proposed approach and the last case is significant only for flat seabed.) The lacunarity is also more accurate than using the simple pixel average, as the latter feature has difficulty distinguishing flat seabed from posidonia. Although our objective is not to perform hard segmentation, the results provide additional evidence that the lacunarity is indeed a useful tool-and a better one than anisotropy and complexity - for characterizing the seafloor (and separating the different seafloor types considered here) in the selected sonar imagery.

To more closely examine the lacunarity of different types of flat seabed, Fig. 9 shows the empirical probability density of lacunarity computed separately from SAS images C (coarse sand), D (mud), and E (fine sand). As can be seen from the figure, there is considerable overlap (i.e., similar lacunarity values) for the mud and fine-sand cases but less overlap for the coarse-sand case, which has slightly higher lacunarity values. The trend observable is that the lacunarity values increase with sediment (scatterer) size, which makes intuitive sense. This might suggest that there is a useful link between lacunarity and sediment size; future work will explore this possibility. 


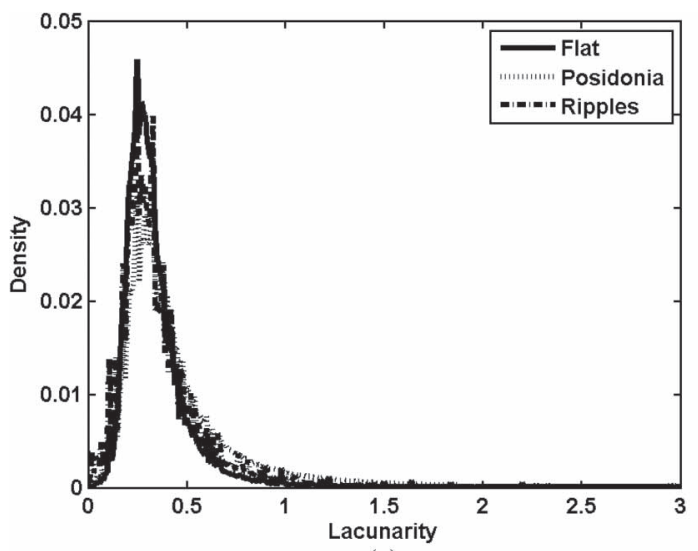

(a)

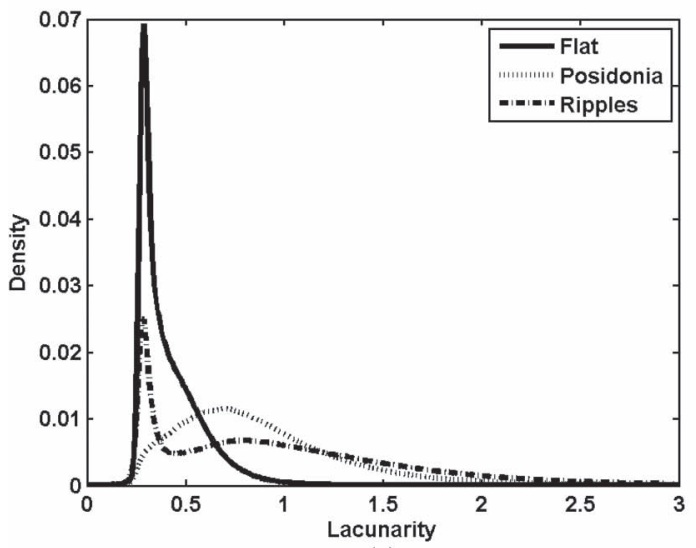

(c)

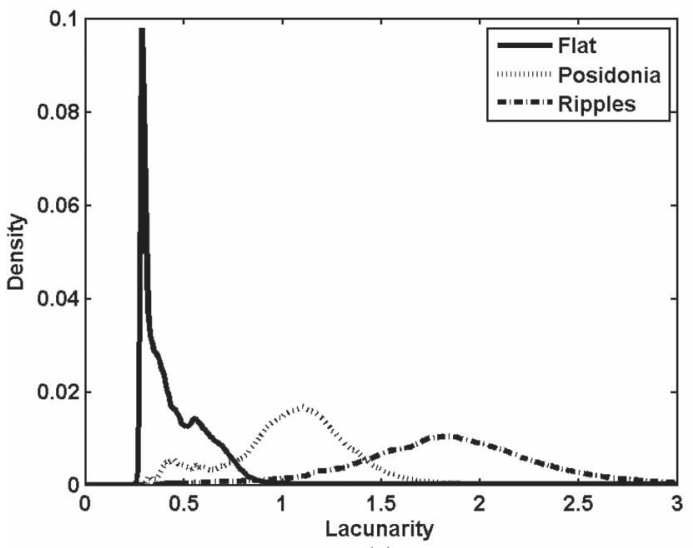

(e)

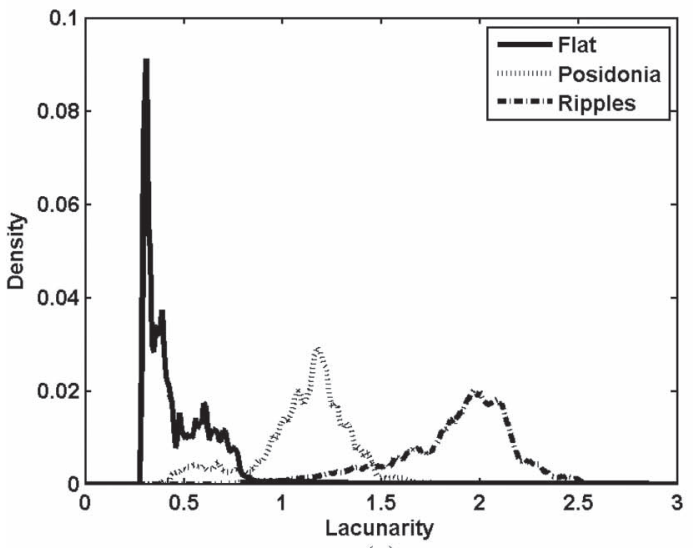

(g)

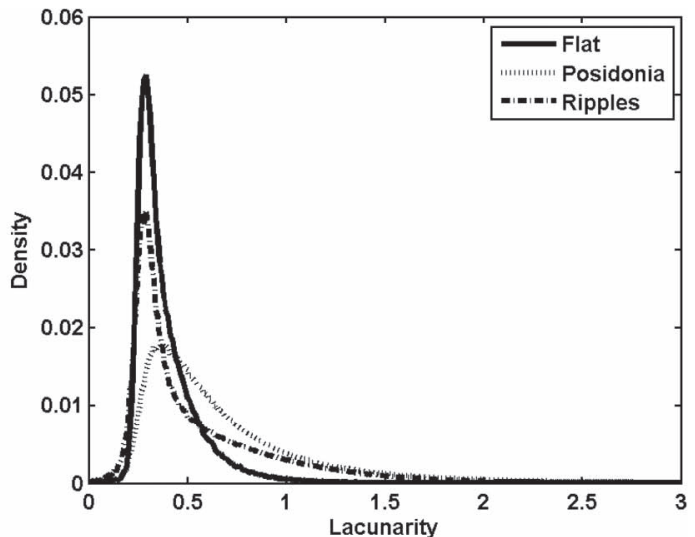

(b)

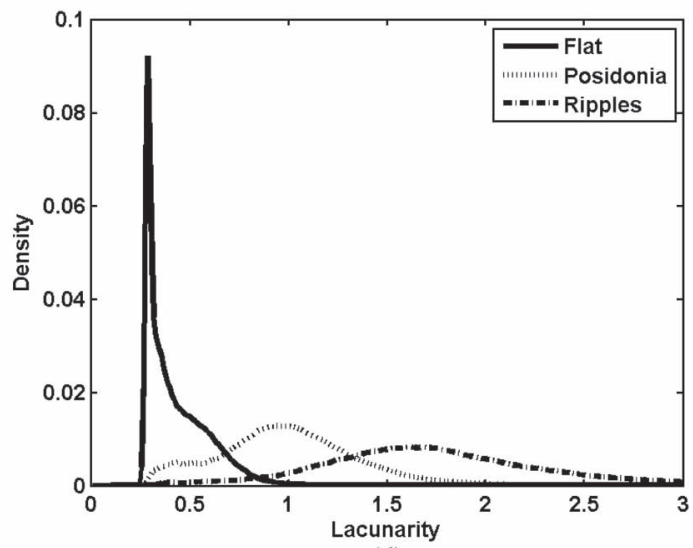

(d)

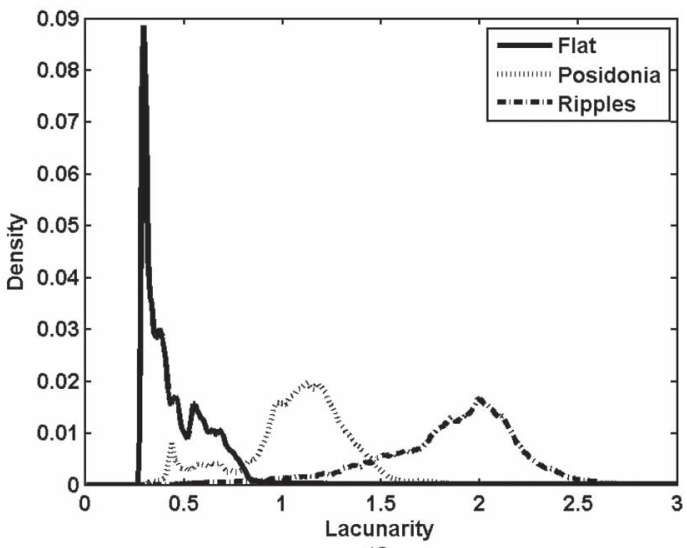

(f)

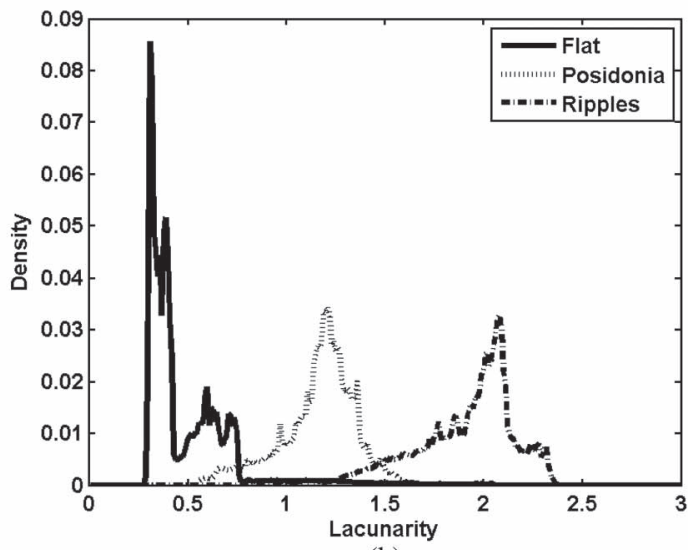

(h)

Fig. 10. Empirical class-conditional probability densities, computed from the five SAS images, of lacunarity when the box used for the calculation has size of (a) $0.125 \mathrm{~m} \times 0.125 \mathrm{~m}$, (b) $0.25 \mathrm{~m} \times 0.25 \mathrm{~m}$, (c) $0.5 \mathrm{~m} \times 0.5 \mathrm{~m}$, (d) $1 \mathrm{~m} \times 1 \mathrm{~m}$, (e) $2 \mathrm{~m} \times 2 \mathrm{~m}$, (f) $4 \mathrm{~m} \times 4 \mathrm{~m}$, (g) $8 \mathrm{~m} \times 8 \mathrm{~m}$, and (h) $16 \mathrm{~m} \times 16 \mathrm{~m}$. 


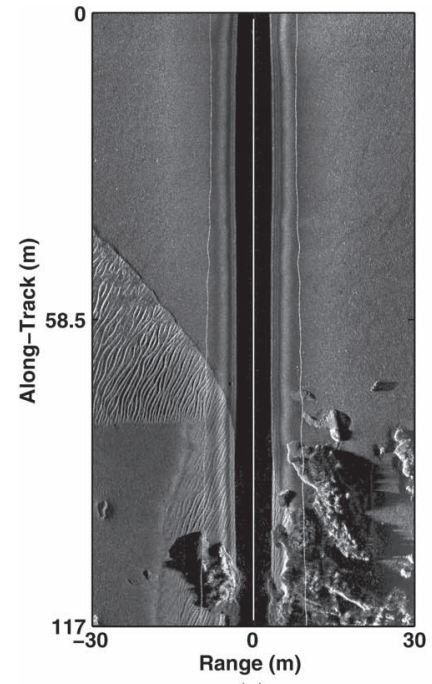

(a)

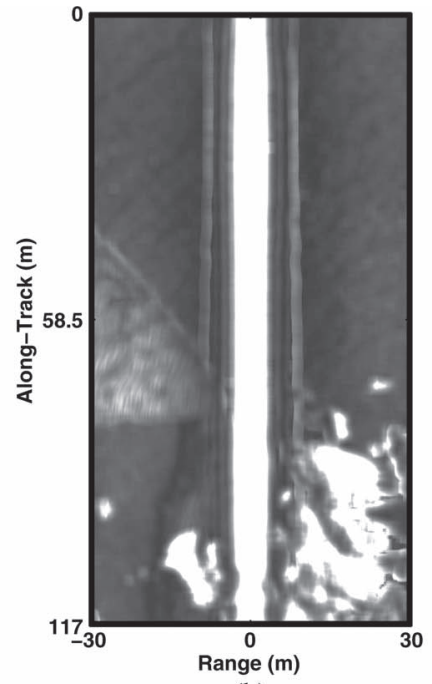

(b)

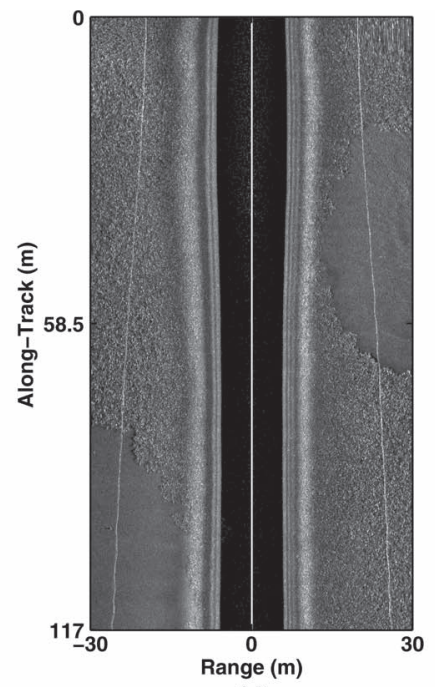

(c)

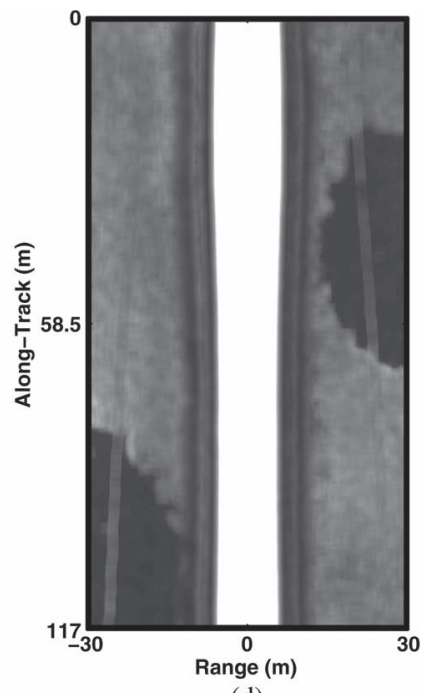

(d)

Fig. 11. (a) Side-scan sonar image $A^{\prime}$ that contains sand ripples and posidonia and the corresponding (b) lacunarity map. (c) Side-scan sonar image $B^{\prime}$ that

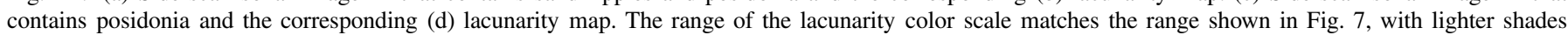
indicating larger values.

As noted previously, the size of the box (i.e., rectangle) used in the lacunarity calculation was chosen to be $2 \mathrm{~m} \times 2 \mathrm{~m}$. Fig. 10 illustrates the effects on the lacunarity values caused by using different box sizes for the calculations. Specifically, the figure shows the empirical class-conditional probability densities, computed from the five SAS images, of lacunarity for the three seafloor types as a function of box size, with sides ranging from 0.125 to $16 \mathrm{~m}$, in powers of 2 .

If the box size is too small, seafloor structures cannot be resolved properly. For example, if the box size is too small in a rippled area, the box (for the lacunarity calculation) might contain only the highlight portion of a ripple (i.e., none of the shadow portion), and the resulting lacunarity would resemble that of flat seabed (because the pixel values' lack of variation would mimic that of flat seabed). Indeed, from the figure, it can be seen that the seafloor classes are indistinguishable when excessively small boxes are used. If the box size is too large, the likelihood of multiple disparate seafloor types falling within a given box increases. (However, the figure suggests that using larger box sizes $(>2 \mathrm{~m})$-for the specific images considered, with relatively few seafloor transitions-would have distinguished the seafloor classes even more successfully, although this was not pursued.)

\section{Applicability to Side-Scan Sonar}

A preliminary assessment of using lacunarity to characterize the seafloor in SAS images has been examined. It is also of interest to determine if lacunarity values are stable not just across different geographical sites but also across different sensors (sonars) operating over different frequencies that produce different resolution imagery. Therefore, we apply the method directly to two lower-resolution side-scan sonar images collected during the same sea experiment from which SAS image B comes.

The images were collected in 2013 near Elba, Italy, with the REMUS AUV, which was equipped with a $900-\mathrm{kHz}$ side-scan sonar. Each image covers approximately $117 \mathrm{~m}$ in the along- track direction and $60 \mathrm{~m}$ in the across-track direction $(30 \mathrm{~m}$ per side, as both port and starboard swaths comprise the image; the nadir gap below the AUV is therefore also present). In the side-scan sonar images, each pixel is assumed to correspond to an area of $11.72 \mathrm{~cm}$ in the along-track direction and 5.859 $\mathrm{cm}$ in the across-track direction (unlike in the SAS images, the resolution is range dependent).

The two side-scan sonar images and their respective lacunarity maps are shown in Fig. 11. As with the SAS images, the lacunarity calculations were based on pixels comprising $2 \mathrm{~m} \times$ $2 \mathrm{~m}$ blocks of seafloor. A visual assessment of the lacunarity suggests the values are consistent with those obtained for SAS images characterized by similar seafloors. Although anecdotal, these results suggest that lacunarity can indeed also be used for side-scan sonar images, which is reasonable since no assumptions (e.g., regarding the pixels' statistical distribution) are being made.

Because the REMUS AUV flies at a lower altitude, shadows tend to cover a larger fraction of the imagery (due to simple geometry). It is interesting to note that the lacunarity is excessively high in the shadow regions of image $A^{\prime}$ (and in the nadir gaps), which is actually consistent with the idea that high lacunarity implies difficult minehunting, as it is impossible to detect targets if they are fully concealed in shadows.

\section{E. Target-Detection Performance Prediction}

The main motivation for characterizing the seafloor was to enable the estimation of minehunting difficulty and, in turn, better predict the target-detection performance that would be achieved at a given site. We next demonstrate how lacunarity can be used in the context of performance prediction.

In each of the eight sea experiments mentioned previously, different groups of man-made targets meant to simulate underwater mines were laid; the targets comprised cylinders, truncated cones, and wedge-shape objects. Targets were located in flat benign seabed, sand ripple fields, and posidonia. Surveys with the MUSCLE AUV were then performed over the target 


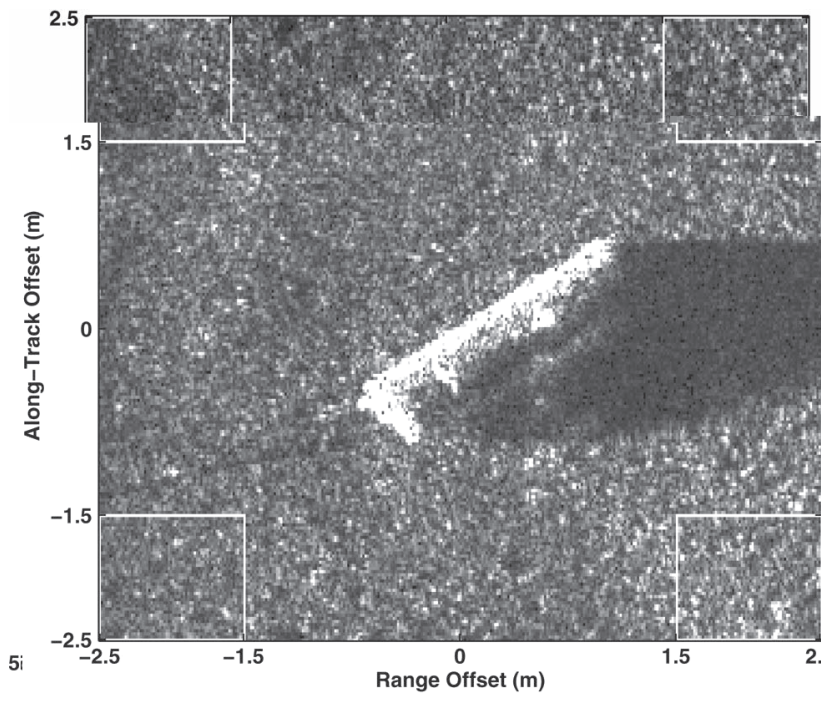

Fig. 12. Example SAS image snippet containing a cylindrical target that also shows the regions (four corner boxes) used to calculate the lacunarity of the seafloor around a target. (For this example, the lacunarity was 0.458 .)

areas (as well as other areas). We apply the target detection algorithm from [11] to all of the SAS images from the eight sea experiments considered. This set constitutes a total of 40774 SAS images, collectively covering over $214 \mathrm{~km}^{2}$ of seafloor, with 2073 target-detection opportunities.

Next, the lacunarity of the seafloor around each target was computed. To prevent the target response from skewing the lacunarity calculation, i.e., to ensure that all pixels used in the calculation correspond to the seafloor, pixels just outside the target region were considered; this is illustrated in more detail by way of an example in Fig. 12. (The total number of pixels used in the lacunarity calculation around the targets is the same as was used previously - here, pixels in four $1 \mathrm{~m} \times 1 \mathrm{~m}$ boxes, compared to one $2 \mathrm{~m} \times 2 \mathrm{~m}$ box.)

Then, the probability of successfully detecting a target as a function of the lacunarity value of the seafloor around the target can be computed. This result is shown in Fig. 13, where a noticeable trend is evident. (The $x$-axis tick labels indicate the edges of the histogram bins used, with larger bins used at the highest lacunarity values to compensate for fewer detection opportunities there.) In general, as the lacunarity increases (from 0 to 1.4), the probability of detection decreases. (Because target detection is easier in sand ripples than posidonia [11], the probability of detection increases again for lacunarity values above 1.4 [cf. Fig. 7(a)].) In other words, when the lacunarity is not very low (e.g., $>0.8$ ), minehunting is more challenging, and performance can be expected to be suffer.

This result makes sense. In flat benign seabed, the lacunarity will be low, and minehunting will be easy. When the lacunarity is higher, the pixel-intensity variation is higher because the seafloor is more complicated, so minehunting will be more challenging. The results provide further support to the claim that the lacunarity is a good surrogate measure for minehunting difficulty.

During an MCM mission, this relationship can be exploited to inform performance prediction models as follows. With a simple lookup table associating lacunarity to the probability of

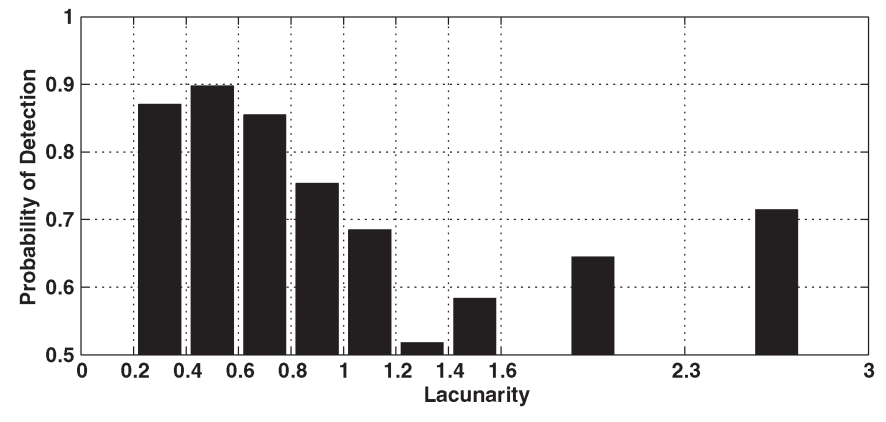

Fig. 13. Probability of detection as a function of the lacunarity of the seafloor around the target.

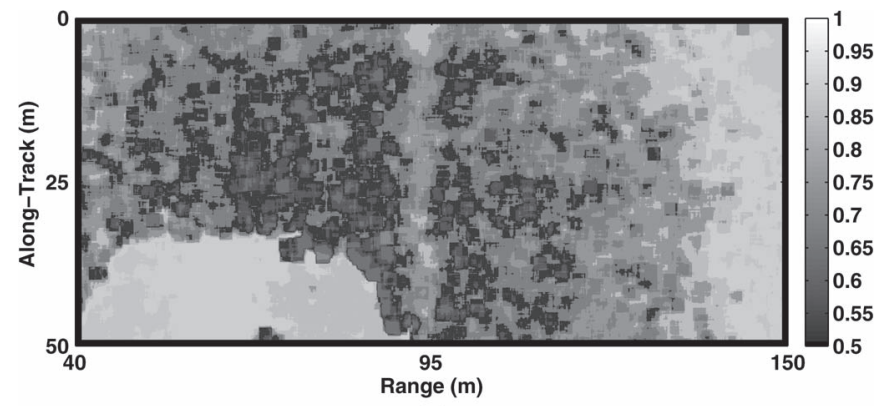

(a)

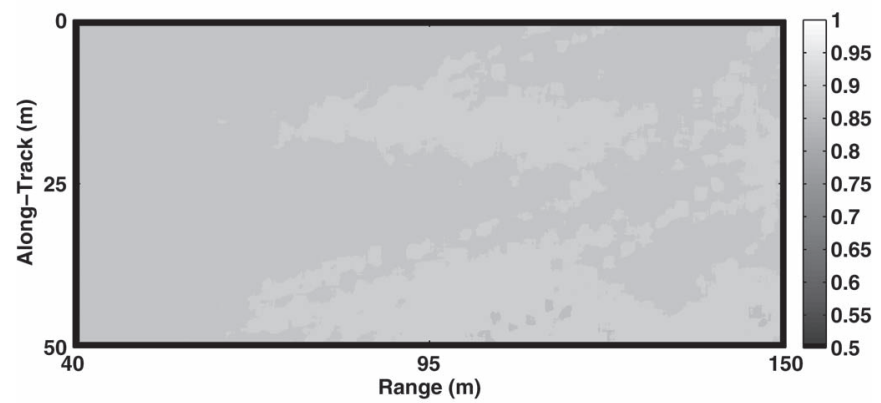

(b)

Fig. 14. Predicted probability of detection map for (a) SAS image B and (b) SAS image E.

detection, as in Fig. 13, one can construct a map providing the predicted probability of successfully detecting a target, if one were present, at each pixel (i.e., seafloor location). This gives a much more nuanced map with finer predictions than if one assigned each pixel to a discrete seafloor class (in which case, the number of unique values in the probability of detection map would be equal to the number of classes). It should be noted that this approach requires historical data (e.g., Fig. 13) expressing the probability of detection for a particular detection algorithm, mine threat, and sensor triplet.

Because the lacunarity can be readily computed on-board an AUV during an MCM mission, in situ estimates of predicted performance at the pixel level can be obtained immediately to guide the remaining survey. Two interesting examples of such performance prediction maps, from using Fig. 13, are shown in Fig. 14.

If a coarser more global assessment of overall predicted performance is desired, the performance prediction maps can be averaged. Doing this for the five SAS images considered results in an overall measure of predicted target-detection performance, shown in Table VII. 
TABLE VII

Predicted Image-Wide Target-Detection Performance

\begin{tabular}{|c||c|}
\hline SAS IMAGE & PROBABILITY OF DETECTION \\
\hline \hline A & $0.699 \pm 0.122$ \\
\hline B & $0.715 \pm 0.126$ \\
\hline C & $0.874 \pm 0.030$ \\
\hline D & $0.870 \pm 0.035$ \\
\hline E & $0.879 \pm 0.013$ \\
\hline
\end{tabular}

It can be observed that the results comport with one's intuition regarding the relative difficulty of performing minehunting in each image; for example, images $\mathrm{A}$ and $\mathrm{B}$, containing mostly ripples and posidonia, respectively, have the lowest overall probability of detection, whereas the three images characterized by benign flat seabed have a high probability of detection with very little variance.

It should be reiterated that these performance prediction maps are obtained without explicitly segmenting the image into discrete classes and without needing any training data for setting lacunarity thresholds.

\section{F. Handling Previously Unseen Seafloor Conditions}

A supervised classification approach would require labeled data to be available from every possible seafloor class in the training stage, but the possibility always exists-especially in MCM operations - that a novel seafloor type will be encountered upon entering new waters. Therefore, it is important that the proposed approach can smoothly handle new previously unseen seafloor conditions.

The key underlying assumption enabling this is as follows: Although the specific seafloor conditions may be novel, the lacunarity values of the new seafloor will be close to those of other types of (familiar) seafloors that impact target-detection performance in a similar manner. (This, in turn, makes use of the fact that detection capability depends mainly on the pixel values near a target's highlight and shadow regions.) This supposition underscores the importance of characterizing the seafloor via a low-dimensional feature space-in this case, 1-D since only lacunarity is used-because the higher the feature dimension, the more likely the assumption would not hold. That is, if the number of features is more than 1 or 2 , it becomes much more likely that a previously unseen seafloor type could map to a region of the high-dimensional feature space unoccupied by any historical data.

To demonstrate the ability of the proposed framework to deal with the scenario of novel seafloor conditions, we consider a new SAS image F, shown in Fig. 15, whose seafloor is characterized by both sand and previously unseen rocky outcroppings.

Although we possessed no historical data of targets in such seafloor conditions, we can still predict the target-detection performance for this new site using Fig. 13. This is possible because the lacunarity values of the rocky outcroppings are similar to other seafloor conditions for which data were available (e.g., the prominent highlights and shadows of sand ripples produce lacunarity values similar to those of the rocky outcroppings). The lacunarity map associated with SAS image $F$ and the resulting predicted probability of detection map are shown in Fig. 16. The average image-wide probability of detection

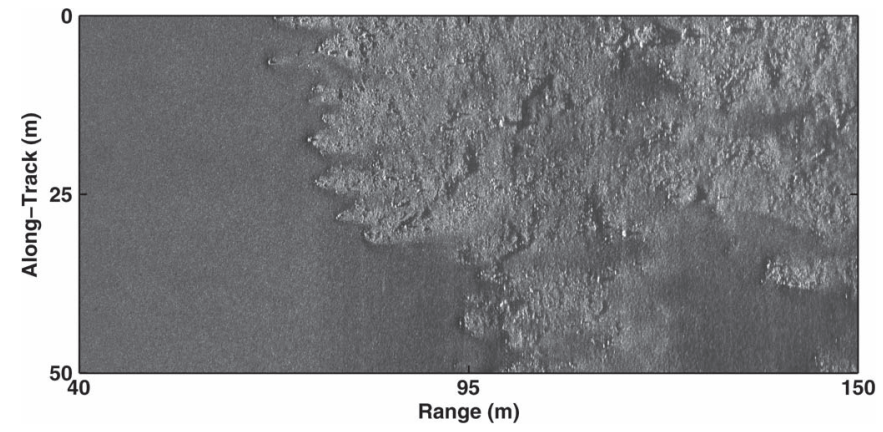

Fig. 15. SAS image F from near Liepāja, Latvia, that has seafloor characterized by both sand and previously unseen rocky outcroppings.

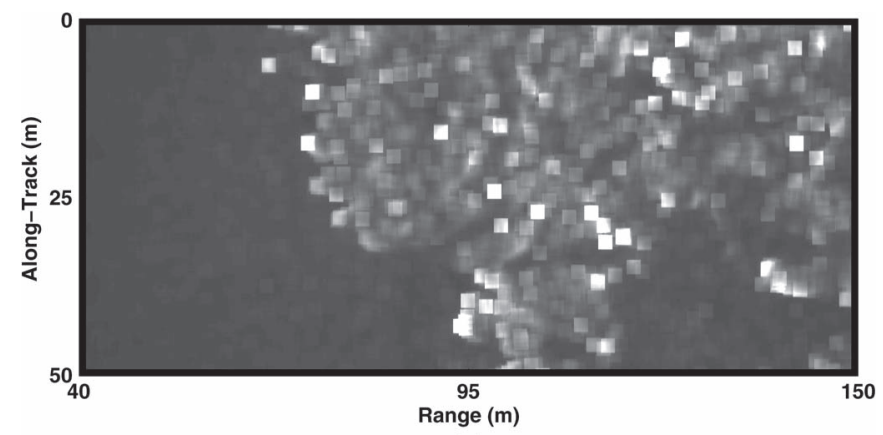

(a)

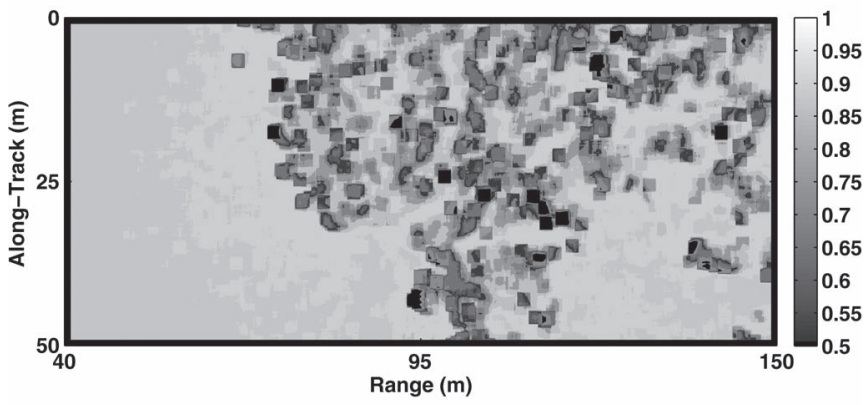

(b)

Fig. 16. For SAS image F, the corresponding (a) lacunarity map and (b) predicted probability of detection map. The range of the lacunarity color scale matches the range shown in Fig. 7, with lighter shades indicating larger values. (In (b), all pure black points correspond to a probability of zero.)

for SAS image F, analogous to those shown in Table VII, was $0.808 \pm 0.137$.

In the event that a new seafloor type is characterized by lacunarity values beyond anything ever before encountered, the predicted probability of detection in those regions would naturally be set to zero, with this clearly signaling that special attention was warranted. In fact, for SAS image F, this occurred in Fig. 16 for a few small isolated regions (totaling 1.21\% of the image), but as can be seen, the method handled this gracefully and did not break down.

\section{CONCLUSION}

A new lacunarity-based approach for characterizing the seafloor of sonar imagery has been introduced. The proposed approach overcomes various limitations that plague other existing techniques, including the need to enumerate the universe of (discrete) seafloor types a priori. The promise of the approach 
was demonstrated on real side-looking sonar imagery measured at various geographical sites in the Baltic and Mediterranean Seas with different seafloor conditions. It was also shown how lacunarity can be computed very quickly and efficiently using integral-image representations. As a result, the approach is appropriate for characterizing seafloor conditions in situ during MCM operations with an AUV. This information can then be used to obtain (delayed) real-time target-detection performance predictions across a site and, in turn, to adapt AUV surveys accordingly so that the most informative data are collected. This capability has already been exploited at sea in an MCM experiment in 2014 using CMRE's MUSCLE AUV.

Future work will use the seafloor lacunarity as a feature to inform an environmentally adaptive target classification algorithm [28]. Additional work will seek to verify the hypothesis that a common lacunarity value generated by different phenomena (e.g., ripples and rocky outcroppings) should indeed map to the same probability of detection.

\section{REFERENCES}

[1] D. Carmichael, L. Linnett, S. Clarke, and B. Calder, "Seabed classification through multifractal analysis of sidescan sonar imagery," Proc. Inst. Elect. Eng. Radar, Sonar Navigat., vol. 143, no. 3, pp. 140-148, Jun. 1996.

[2] P. Blondel, L. Parson, and V. Robigou, "TexAn: Textural analysis of sidescan sonar imagery and generic seafloor characterisation," in Proc. IEEE OCEANS, 1998, vol. 1, pp. 419-423.

[3] S. Reed, I. Ruiz, C. Capus, and Y. Petillot, "The fusion of large scale classified sidescan sonar image mosaics," IEEE Trans. Image Process., vol. 15, no. 7, pp. 2049-2060, Jul. 2006.

[4] D. Williams, "Bayesian data fusion of multiview synthetic aperture sonar imagery for seabed classification," IEEE Trans. Image Process., vol. 18, no. 6, pp. 1239-1254, Jun.2009.

[5] I. Karoui, R. Fablet, J. Boucher, and J. Augustin, "Seabed segmentation using optimized statistics of sonar textures," IEEE Trans. Geosci. Remote Sens., vol. 47, no. 6, pp. 1621-1631, Jun. 2009.

[6] D. Abraham and A. Lyons, "Novel physical interpretations of K-distributed reverberation," IEEE J. Ocean. Eng., vol. 27, no. 4, pp. 800-813, Oct. 2002.

[7] D. Abraham and A. Lyons, "Reliable methods for estimating K-distribution shape parameter," IEEE J. Ocean. Eng., vol. 35, no. 2, pp. 288-302, Apr. 2010.

[8] J. Cobb, K. Slatton, and G. Dobeck, "A parametric model for characterizing seabed textures in synthetic aperture sonar images," IEEE J. Ocean. Eng., vol. 35, no. 2, pp. 250-266, Apr. 2010.

[9] O. Daniell, Y. Petillot, and S. Reed, "Unsupervised sea-floor classification for automatic target recognition," in Proc. Int. Conf. Underwater Remote Sens., 2012, pp. 136-145.

[10] E. Fakiris, D. Williams, M. Couillard, and W. Fox, "Sea-floor acoustic anisotropy and complexity assessment towards prediction of ATR performance," in Proc. Int. Conf. Exhib. Underwater Acoust., 2013, pp. $1277-1284$.

[11] D. Williams, "Fast target detection in synthetic aperture sonar imagery: A new algorithm and large-scale performance analysis," IEEE J. Ocean. Eng., vol. 40, no. 1, pp. 71-92, Jan. 2015.

[12] G. Henebry and H. Kux, "Lacunarity as a texture measure for SAR imagery," Remote Sens., vol. 16, no. 3, pp. 565-571, 1995.

[13] A. Solberg and A. Jain, "Texture fusion and feature selection applied to SAR imagery," IEEE Trans. Geosci. Remote Sens., vol. 35, no. 2, pp. 475-479, Mar. 1997.

[14] G. Du and T. Yeo, "A novel lacunarity estimation method applied to SAR image segmentation," IEEE Trans. Geosci. Remote Sens., vol. 40, no. 12, pp. 2687-2691, Dec. 2002.

[15] R. Dekker, "Texture analysis and classification of ERS SAR images for map updating of urban areas in The Netherlands," IEEE Trans. Geosci. Remote Sens., vol. 41, no. 9, pp. 1950-1958, Sep. 2003.

[16] D. Greenhill, L. Ripke, A. Hitchman, G. Jones, and G. Wilkinson, "Characterization of suburban areas for land use planning using landscape ecological indicators derived from Ikonos-2 multispectral imagery," IEEE Trans. Geosci. Remote Sens., vol. 41, no. 9, pp. 2015-2021, Sep. 2003.
[17] S. Myint, C. Giri, L. Wang, Z. Zhu, and S. Gillette, "Identifying mangrove species and their surrounding land use and land cover classes using an object-oriented approach with a lacunarity spatial measure," GISci. Remote Sens., vol. 45, no. 2, pp. 188-208, 2008.

[18] J. Weishampel, J. Blair, R. K. R. Dubayah, and D. Clark, "Volumetric lidar return patterns from an old-growth tropical rainforest canopy," Int. J. Remote Sens., vol. 21, no. 2, pp. 409-415, 2000.

[19] A. Einstein, H. Wu, and J. Gil, "Self-affinity and lacunarity of chromatin texture in benign and malignant breast epithelial cell nuclei," Phys. Rev. Lett., vol. 80, no. 2, pp. 397-400, 1998.

[20] N. Valous, D. Sun, P. Allen, and F. Mendoza, "The use of lacunarity for visual texture characterization of pre-sliced cooked pork ham surface intensities," Food Res. Int., vol. 43, no. 1, pp. 387-395, 2010.

[21] R. Kessel, "Using sonar speckle to identify regions of interest and for mine detection," Proc. AeroSense, 2002, pp. 440-451.

[22] J. Nelson and N. Kingsbury, "Fractal dimension, wavelet shrinkage and anomaly detection for mine hunting," IET Signal Process., vol. 6, no. 5, pp. 484-493, 2012.

[23] J. Nelson and V. Krylov, "Textural lacunarity for semi-supervised detection in sonar imagery," IET Radar, Sonar Navigat., vol. 8, no. 6, pp. 616-621, Jul. 2014.

[24] L. Sha and L. Nolte, "Effects of environmental uncertainties on sonar detection performance prediction," J. Acoust. Soc. Amer, vol. 117, no. 4, pp. 1942-1953, Apr. 2005.

[25] D. Abraham, "Detection-threshold approximation for non-Gaussian backgrounds," IEEE J. Ocean. Eng., vol. 35, no. 2, pp. 355-365, Apr. 2010.

[26] C. Ratto, P. Torrione, and L. Collins, "Exploiting ground-penetrating radar phenomenology in a context-dependent framework for landmine detection and discrimination," IEEE Trans. Geosci. Remote Sens., vol. 49, no. 5, pp. 1689-1700, May 2011.

[27] C. Ratto, K. Morton, L. Collins, and P. Torrione, "Bayesian contextdependent learning for anomaly classification in hyperspectral imagery," IEEE Trans. Geosci. Remote Sens., vol. 52, no. 4, pp. 1969-1981, Apr. 2014.

[28] D. Williams and E. Fakiris, "Exploiting environmental information for improved underwater target classification in sonar imagery," IEEE Trans. Geosci. Remote Sens., vol. 52, no. 10, pp. 6284-6297, Oct. 2014.

[29] B. Mandelbrot, The Fractal Geometry of Nature. New York, NY, USA: Macmillan, 1983, vol. 173.

[30] R. Plotnick, R. Gardner, W. Hargrove, K. Prestegaard, and M. Perlmutter, "Lacunarity analysis: A general technique for the analysis of spatial patterns," Phys. Rev. E, vol. 53, no. 5, pp. 5461-5468, 1996.

[31] P. Viola and M. Jones, "Robust real-time object detection," Int. J. Comput. Vis., vol. 57, no. 2, pp. 137-154, 2004.

[32] C. Allain and M. Cloitre, "Characterizing the lacunarity of random and deterministic fractal sets," Phys. Rev. A, vol. 44, no. 6, pp. 3552-3558, 1991.

[33] R. Plotnick, R. Gardner, and R. O'Neill, "Lacunarity indices as measures of landscape texture," Landscape Ecol., vol. 8, no. 3, pp. 201-211, 1993.

[34] G. Facciolo, N. Limare, and E. Meinhardt, "Integral images for block matching," Image Process. Line, vol. 4, pp. 344-369, 2014.

[35] M. Hayes and P. Gough, "Broad-band synthetic aperture sonar," IEEE J. Ocean. Eng., vol. 17, no. 1, pp. 80-94, Jan. 1992.

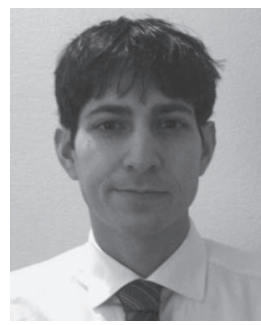

David P. Williams received the B.S.E. (magna cum laude), M.S., and Ph.D. degrees in electrical and computer engineering from Duke University, Durham, NC, USA, in 2002, 2003, and 2006, respectively.

He was the recipient of a James B. Duke Graduate Fellowship and a National Defense Science and Engineering Graduate Fellowship. Since 2007, he has been with the NATO Science and Technology Organization, Centre for Maritime Research and Experimentation (formerly NATO Undersea Research Centre), in La Spezia, Italy. His research interests are in the fields of machine learning, pattern recognition, and mine countermeasures. 